\title{
Redesigning journal club in residency
}

This article was published in the following Dove Press journal:

Advances in Medical Education and Practice

27 May 2016

Number of times this article has been viewed

\section{Morhaf Al Achkar}

Department of Family Medicine, Indiana University, Indianapolis, IN, USA
Correspondence: Morhaf Al Achkar Department of Family Medicine, Indiana University, 1520 North Senate Avenue, Indianapolis, IN 46205, USA

Tel + I 3179628893

Fax + I 3179621048

Email alachkar@iupui.edu
Abstract: The gap between production and implementation of knowledge is the main reason for the suboptimal quality of health care. To eliminate this gap and improve the quality of patient care, journal club (JC) in graduate medical education provides an opportunity for learning the skills of evidence-based medicine. JC, however, continues to face many challenges mainly due to poorly defined goals, inadequate preparation, and lack of interest. This article presents an innovative model to prepare and present JC based on three pillars: dialogical learning through group discussion, mentored residents as peer teachers, and including JC as part of a structured curriculum to learn evidence-based medicine. This engaging model has the potential to transform JC from a moribund session that is daunting for residents into a lively discussion to redefine clinical practice using the most current evidence.

Keywords: journal club, residents, peer teaching, evidence-based medicine, dialogical learning

\section{Introduction}

The gap between the production and implementation of knowledge is the main reason for the suboptimal quality of health care. To improve the quality of care through eliminating this gap, evidence-based medicine (EBM) has emerged as a priority. ${ }^{1} \mathrm{EBM}$ is defined as "the conscientious, explicit, and judicious use of current best evidence in making decisions about the care of individual patients". ${ }^{2}$ Despite its importance for enhancing physicians' life-long learning and patients' outcomes, EBM has continued to be suboptimally practiced because of various barriers. ${ }^{3}$ Among the top barriers to EBM in primary care are inadequate skills and lack of training. ${ }^{1}$

Journal club (JC), a common venue to learn EBM skills, has been used across graduate medical education. ${ }^{4} \mathrm{JC}$ is described as "a group of individuals who meet regularly to discuss critically the clinical applicability of articles in the current medical journals". ${ }^{5}$ In JC discussions, published articles are critically evaluated to arrive at fair interpretations of the study findings independent of the author's conclusions. ${ }^{6}$ Early JC was closer to a social gathering in a relaxed atmosphere than to mandated educational meetings for the completion of specific training. However, over the past century, with the emerging need to stay current with the growing number of publications, JC has increased in popularity, taken different forms, and emerged as a more formal learning venue. ${ }^{7,8}$

In spite of the wide incorporation of JC in graduate medical education, it is, the literature shows, rarely successful in maintaining the residents' interest and achieving 
the desired educational objectives. ${ }^{9}$ Many residents see presenting at JC as a daunting process for which undergraduate medical education never adequately prepared them. ${ }^{10}$ Class discussion in JC is often inhibited because of participants' unfamiliarity with the journals' format and writing styles as well as the lack of the knowledge needed to critically read an article. ${ }^{11}$

This article presents an innovative model for the implementation of an engaging and effective JC in residency.

\section{JC at a family medicine residency}

In 2012, a university-based family medicine residency program restructured its JC to build a model that engages residents in a peer-teaching experience. This redesign took place to align JC learning with principles of adult education. The author of this article mentors the residents and helps prepare them to lead large-group discussion, fostering collaborative learning. This model revolves around three main pillars: 1) dialogical learning; 2) mentored residents as peer teachers; and 3) including JC as part of a structured curriculum to learn EBM. JC takes place once a month during the regular protected didactic time, which is on Thursday afternoons. Attendance at JC is mandatory, and the majority of residents attend the learning sessions unless they are on night calls or covering critical services.

\section{Selecting the articles}

The residents identify articles and discuss their selections with the mentoring faculty. A repository of recently published articles, maintained by the academic chief resident and the mentoring faculty, is also available. The selected papers are judged based on five criteria: 1) the significance of the research question; 2) the importance of the study design; 3 ) the impact of the findings on clinical practice; 4) the applicability of findings to primary care; and finally, 5) interest in the topic among the presenting residents. The selected article and a specific critical appraisal sheet are then shared with the residents a week prior to the date of the JC. The residents are also sent a reminder to read the articles 2 days prior to the date of the JC.

The articles are selected from high-impact journals such as the New England Journal of Medicine, the Journal of the American Medical Association, the British Medical Journal, and the Annals of Internal Medicine. The articles cover a wide scope of research questions, including therapy, diagnosis, prognosis, etiology, and economical analysis. The articles also illustrate a variety of research designs, including metaanalyses, randomized controlled trials, cohort studies, and case-control studies. The study designs in JC complement the longitudinal EBM curriculum that is delivered as a 1-hour workshop bimonthly. During these workshops, the residents are instructed on the main concepts of critical appraisal to build the foundational knowledge necessary for their own critical reading and appraisal of articles of different designs.

\section{Preparing residents for presentation}

The faculty mentor prepares the residents to lead a largegroup discussion through both ensuring the mastery of the critical appraisal of the identified article and through planning the interactive discussion. In each JC session, four residents are scheduled to lead the discussion of two articles. The presenting residents critically appraise the assigned article on their own prior to meeting with the mentoring faculty a few days before the JC session. During this meeting, the resident and the faculty reflect on the importance of the article's topic and then identify the research question and study design before they critically appraise the study. Finally, a simplified PowerPoint presentation is prepared to map the discussion that includes the following six elements: 1) the importance and background of the topic; 2) the research question and study design; 3) patient, intervention, comparison, and outcomes (if applicable); 4) criteria for validity of the study design; 5) the results of the study; and finally, 6) the applicability of the study and its impact on practice.

\section{JC discussion}

With the exception of the background section, which is delivered via an engaging slide presentation, the rest of the JC elements are delivered in a large-group discussion where the presenting residents ask a question and allow time for the participants to identify the answers in the handed-out articles. The background information is used to elicit interest through highlighting the prevalence of the problem, the importance of the condition in patients' lives, and the limitations of knowledge that led to the article's research question. Then, the research question is defined in the population of the study, intervention, comparison, and outcomes (PICO) format, if applicable. The facilitator then explores the validity criteria. For example, in questions about therapy, these criteria will include the adequacy of the randomization process and its concealment, the similarity between the groups, the completion of follow-up and the sufficiency of its duration, the intention to treat, and the equality of treatment of the groups in the different arms of the study. For each point, the presenter asks the audience to identify whether the ideal way of conducting the study was done. If the study did not meet 
the specific criteria, the residents discuss whether that would pose a significant threat to validity.

Discussing the results takes place afterward, utilizing the figures, graphs, and tables, and through assessing the statistical significance. The clinical significance of the results is highlighted thereafter. Finally, the discussion moves to the applicability of the study findings in the residents' clinical setting. Questions about the availability of the interventions, the patient's values and preferences, and the barriers to utilizing the study's proposed new findings are then proposed. This is usually followed by an open discussion on how the particular study would change how the participants will be practicing. This discussion takes the form of a heated debate about the pros and cons of the study between its opponents and proponents. At the end of the presentations, the participating audience members vote for the best presentation using a voting card that asks them to rate the quality of the led discussion across the three areas of the critical appraisal. The winning team gets a coffee gift card of $\$ 15$. Table 1 includes practical recommendations that can be followed by residencies interested in implementing JC.

\section{Discussion}

JC is an integral part of graduate medical education training and has long been advocated for as a bridge connecting research and practice. We are proposing a novel model for the implementation of JC with three pillars: 1) residents as mentored peer teachers; 2) dialogic learning in large-group discussion; and 3) an integrated longitudinal EBM curriculum. In this article, we aimed to describe the format of JC in our residency with the hope that graduate medical educators will find the experience useful to replicate.

Since its inception over a century ago, JC has taken many forms, with varied successes and limitations. Our model for $\mathrm{JC}$ is faithful to the main purposes that Sir William Osler established his first JC for, namely, "distributing periodicals" to teach physicians the critical analysis of research work. ${ }^{12}$ In addition, our model is in line with adult learning theory, as it engages learners in a relaxed environment where they participate in planning the activities, teach one another, and take charge of identifying their learning goals in a learning experience that is relevant and meaningful. ${ }^{13}$

Selecting the articles can be arguably considered the most important step toward a successful JC. ${ }^{14}$ Good-quality peer-reviewed articles can potentially change or improve practice. Engaging the residents in the process of selecting the articles was a step that the residents requested and proved fruitful in enhancing ownership and participation. Engaging
Table I Practical recommendations for a successful JC

I. JC should be part of a structured curriculum to learn EBM in residency.

2. Mentoring the residents to prepare and lead JC discussion should be considered to enhance the quality of the learning and teaching.

3. Group discussion rather than didactic presentation for JC might be of better educational value.

4. Engaging the residents in selecting the articles seems to enhance their ownership and improve their interest.

5. Following the same systematic approach in every JC could help make the presentation easier to follow and less daunting for residents.

Abbreviations: JC, journal club; EBM, evidence-based medicine.

the academic chief resident not only provides a voice for the residents in directing JC but also serves to both prepare the chief for future academic roles and help with the workload of the mentoring faculty. The structured curriculum to teach EBM serves to prepare the residents with the foundational knowledge and skills needed to conduct critical appraisal. The supportive mentorship and guidance from a devoted faculty also ensures the maintenance of the structure and systematic presentation without necessarily limiting the abilities of the resident to be creative or innovative in his or her presentation.

This model of presenting JC has the potential to transform JC from a moribund session to a lively discussion that not only brings research into practice but also gives residents lifelong learning skills that could continue to be useful throughout their careers.

\section{Acknowledgment}

This work would not have been possible without the support of the Indiana University Health Values Fund Grant (VFE-319).

\section{Disclosure}

The author reports no conflicts of interest in this work.

\section{References}

1. Sadeghi-Bazargani H, Tabrizi JS, Azami-Aghdash S. Barriers to evidence-based medicine: a systematic review. J Eval Clin Pract. 2014;20(6):793-802.

2. Sackett DL, Rosenberg WM, Gray JA, Haynes RB, Richardson WS. Evidence based medicine: what it is and what it isn't. $B M J$. 1996;312(7023):71-72.

3. Maggio LA, Kung JY. How are medical students trained to locate biomedical information to practice evidence-based medicine? A review of the 2007-2012 literature. J Med Libr Assoc. 2014;102(3):184-191.

4. Webber E, Saysana M, McKenna M. Journal club blog for faculty paediatricians. Med Educ. 2013;47(11):1128.

5. Arif SA, Gim S, Nogid A, Shah B. Journal clubs during advanced pharmacy practice experiences to teach literature-evaluation skills. $A m$ J Pharm Educ. 2012;76(5):88.

6. Baker JD. Journal club as a resource for practice. AORN J. 2013;98(2): 102-106. 
7. Millichap JJ, Goldstein JL. Neurology journal club: a new subsection. Neurology. 2011;77(9):915-917.

8. Linzer M. The journal club and medical education: over one hundred years of unrecorded history. Postgrad Med J. 1987;63(740):475-478.

9. Tam KW, Tsai LW, Wu CC, Wei PL, Wei CF, Chen SC. Using vote cards to encourage active participation and to improve critical appraisal skills in evidence-based medicine journal clubs. J Eval Clin Pract. 2011;17(4):827-831.

10. Bowles PF, Marenah K, Ricketts DM, Rogers BA. How to prepare for and present at a journal club. Br J Hosp Med (Lond). 2013;74(10): $\mathrm{C} 150-\mathrm{C} 152$.
11. McDonough V. Improving journal club: increasing student discussion and understanding of primary literature in molecular biology through the use of dialectical notes. Biochem Mol Biol Educ. 2012;40(5):330-332.

12. Amadio J. Introducing neurosurgery's journal club. Neurosurgery. 2012;71(5):907-908.

13. Hartzell JD, Veerappan GR, Posley K, Shumway NM, Durning SJ. Resident run journal club: a model based on the adult learning theory. Med Teach. 2009;31(4):e156-e161.

14. Judd S, Antaki F. Approach to presenting a clinical journal club. Gastroenterology. 2014;146(7):1591-1593.

\section{Publish your work in this journal}

Advances in Medical Education and Practice is an international, peerreviewed, open access journal that aims to present and publish research on Medical Education covering medical, dental, nursing and allied health care professional education. The journal covers undergraduate education, postgraduate training and continuing medical education including emerging trends and innovative models linking education, research, and health care services. The manuscript management system is completely online and includes a very quick and fair peer-review system. Visit http://www.dovepress.com/testimonials.php to read rea quotes from published authors.

Submit your manuscript here: http://www.dovepress.com/advances-in-medical-education-and-practice-journal 